\title{
Flutter auricular: Resultados inmediatos y alejados de la fulguración con radiofrecuencia del istmo cavo-tricuspídeo
}

\author{
Carlos Almendares M, Patricia Frangini S, \\ Ismael Vergara S, Mariana Baeza La , Rolando González A. \\ Results of radiofrequency ablation of \\ the inferior vena cava-tricuspid valve \\ isthmus for atrial flutter
}

Background: Radiofrequency ablation of the inferior vena cava-tricuspid valve isthmus relieves atrial flutter in 95\% of cases. Aim: To evaluate the long term results of radiofrequency ablation of the inferior vena cava-tricuspid valve isthmus in atrial flutter. Material and methods: Retrospective review of 86 patients with common atrial flutter, treated with radiofrequency ablation of the isthmus, while in sinus rhythm or flutter. Patients were contacted by telephone for a new clinical and electrocardiographic assessment, three to 40 months after the procedure. Results: Of all the patients treated, five died and five were lost from follow up, thus 76 patients (mean age 56 years, 58 males) were contacted for reassessment. At the moment of the procedure, $51 \%$ had an underlying cardiac disease and $25 \%$ had high blood pressure. All referred palpitations, $25 \%$ had dyspnea, $84 \%$ were receiving antiarrhythmic drugs and 33\% were on oral anticoagulants. Flutter was paroxystic in $83 \%$ and chronic in $17 \%$. Fulguration was successful in all patients; one patient presented a high grade atrioventricular block as a complication of the procedure. At reassessment, $82 \%$ of patients were in sinus rhythm, $16 \%$ had atrial fibrillation and $2 \%$, an atypical flutter. Conclusions: Radiofrequency fulguration is a safe and effective treatment of atrial flutter (Rev Méd Chile 2005; 133: 159-66).

(Key Words: Arrhythmia; Atrial flutter; Radiofrequency catheter ablation)

Recibido el 31 de marzo 2004. Aceptado en versión corregida el 4 de noviembre 2004. Laboratorio de Electrofisiología Cardíaca. Departamento de Enfermedades Cardiovasculares. Facultad de Medicina. Pontificia Universidad Católica de Chile, Santiago-Chile.

aEnfermera Universitaria.

Correspondencia a: Dr. Rolando González A. Marcoleta

$3675^{\circ}$ piso. Fono: 6333171. Fax: 6333171.

E-mail: rgonzale@med.puc.cl 
$E^{1}$ flutter auricular típico (FL) es una arritmia frecuente, que ocurre tanto en corazones sanos como en corazones enfermos y cuyo tratamiento clínico es complejo, dada su alta recurrencia y refractariedad al tratamiento farmacológico. Ésta se caracteriza por tener un ciclo auricular casi constante, con frecuencias entre 250 y 350 latidos por minuto. La conducción aurículo-ventricular suele ser con bloqueo 2 por 1 .

Esta arritmia es causada por un circuito de macrorreentrada en la aurícula derecha $(\mathrm{AD})$ que tiene una zona de conducción protegida y obligada a través del istmo cavo-tricuspídeo (ICT). Éste está delimitado posteriormente por la vena cava inferior, anteriormente por el anillo tricuspídeo, hacia medial por el ostium del seno coronario y el reborde de Eustaquio y hacia lateral por la crista terminalis (Figura 1). En su variedad más frecuente el circuito de FL tiene sentido antihorario. En este caso, el impulso eléctrico recorre en forma ascendente el septum interauricular hasta el techo de la $\mathrm{AD}$ y baja por la crista terminalis, activando la $\mathrm{AD}$ en sentido antihorario y desde allí pasa por el ICT, donde se enlentece transitoriamente para llegar nuevamente hasta el septum interauricular. Esto genera un electrocardiograma característico, con ondas de FL negativas en las derivaciones II, III y AVF y deflexiones auriculares positivas en V1.

El impulso eléctrico también puede recorrer el mismo camino pero en sentido contrario (en el sentido de los punteros del reloj): asciende por la cara lateral de la $\mathrm{AD}$ y desciende por el septum interauricular. En este caso el FL también será istmo dependiente, pero su apariencia en el electrocardiograma de superficie será diferente pues las ondas de FL serán positivas en la pared inferior (derivaciones II, III y AVF) y negativas en V1. Este es el denominado flutter típico inverso y es menos frecuente que el anterior.

Existen circuitos no istmo dependientes descritos en la aurícula izquierda, alrededor del seno coronario y en otros sitios menos definidos, conformando los llamados flutter atípicos ${ }^{1,2}$.

La interrupción de la conducción del impulso eléctrico en el ICT, mediante radiofrecuencia (RF) impide que el circuito de FL típico se complete, con lo cual se reestablece y mantiene ritmo sinusal $^{3-5}$. Dada la alta recurrencia del FL y su refractariedad al tratamiento farmacológico, la interrupción de la conducción en el ICT es, en la

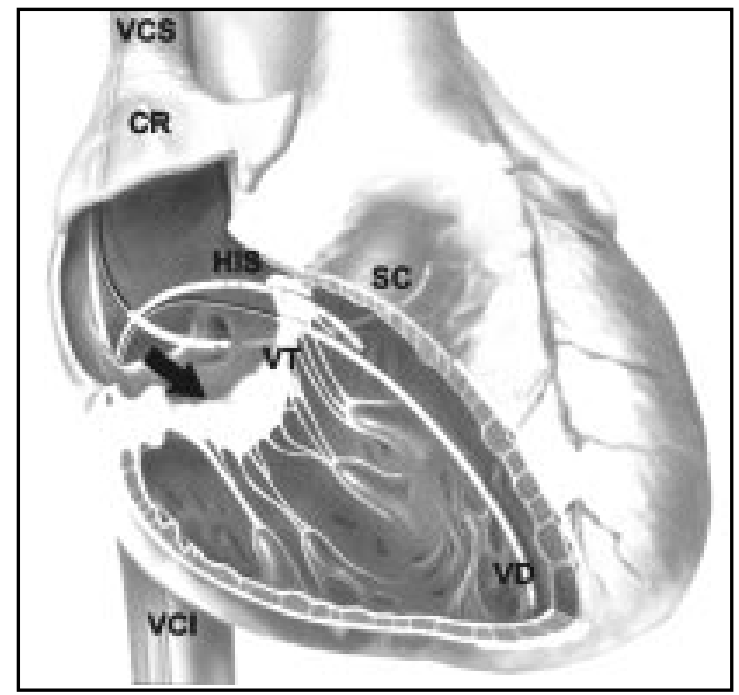

Figura 1. Corazón en que se ha abierto la aurícula y el ventrículo derecho. Se observa un catéter duodecapolar ubicado en crista terminalis (CR) por la cara lateral de la aurícula derecha, un catéter decapolar en seno coronario (SC) y catéteres cuadripolares en His (HIS) y ápex de ventrículo derecho (VD). La flecha está ubicada en el istmo cavo-tricuspídeo. VT: Válvula tricúspide.

actualidad, el tratamiento de elección y definitivo a partir del primer episodio de esta arritmia.

El propósito del presente trabajo es mostrar los resultados inmediatos y alejados en 86 pacientes consecutivos con FL tratados con fulguración del ICT en nuestro laboratorio.

\section{MATERIAL Y MÉTODO}

Pacientes. Se realizó una revisión retrospectiva de los estudios electrofisiológicos realizados en el laboratorio de Electrofisiología Cardíaca del Hospital Clínico de la Universidad Católica entre agosto de 1999 y septiembre de 2002. Se incluyeron 86 pacientes consecutivos con FL referidos para fulguración del ICT con RF. El análisis final se realizó con 76 pacientes, dado que 5 fallecieron y 5 fueron perdidos de seguimiento.

Estudio electrofisiológico. El estudio electrofisiológico (EEF) se realizó bajo sedación con midazolam, morfina y anestesia local. Mediante técnica 
de Seldinger por vía venosa femoral derecha, se posicionaron catéteres multipolares en región de la crista terminalis y región del His. Por vía venosa yugular interna derecha se posicionó catéter decapolar en seno coronario.

En un polígrafo Prucka 4.01, se registraron las señales del electrocardiograma de superficie y de los trazados endocavitarios en condición basal, durante estimulación auricular, ventricular y durante infusión de isoproterenol. La estimulación se realizó con un estimulador Medtronic 5328.

En los pacientes que ingresaron al laboratorio en FL, se documentó la existencia de éste mediante registro de electrocardiograma de superficie y señales endocavitarias que demostraron la existencia de una arritmia auricular con ciclo de $200 \pm$ $20 \mathrm{~ms}$, con secuencia de activación típica. En el caso de FL antihorario, éste se caracteriza por activación cráneo-caudal a través de la crista terminalis, látero-medial por el ICT y caudocraneal por el septum interauricular (Figura 2). En algunos de estos pacientes, se confirmó el mecanismo de reentrada mediante estimulación auricular rápida y en otros se procedió a fulgurar de inmediato la región del ICT.

En los pacientes que ingresaron al laboratorio en ritmo sinusal, se procedió a inducir el FL mediante estimulación auricular, para confirmar el

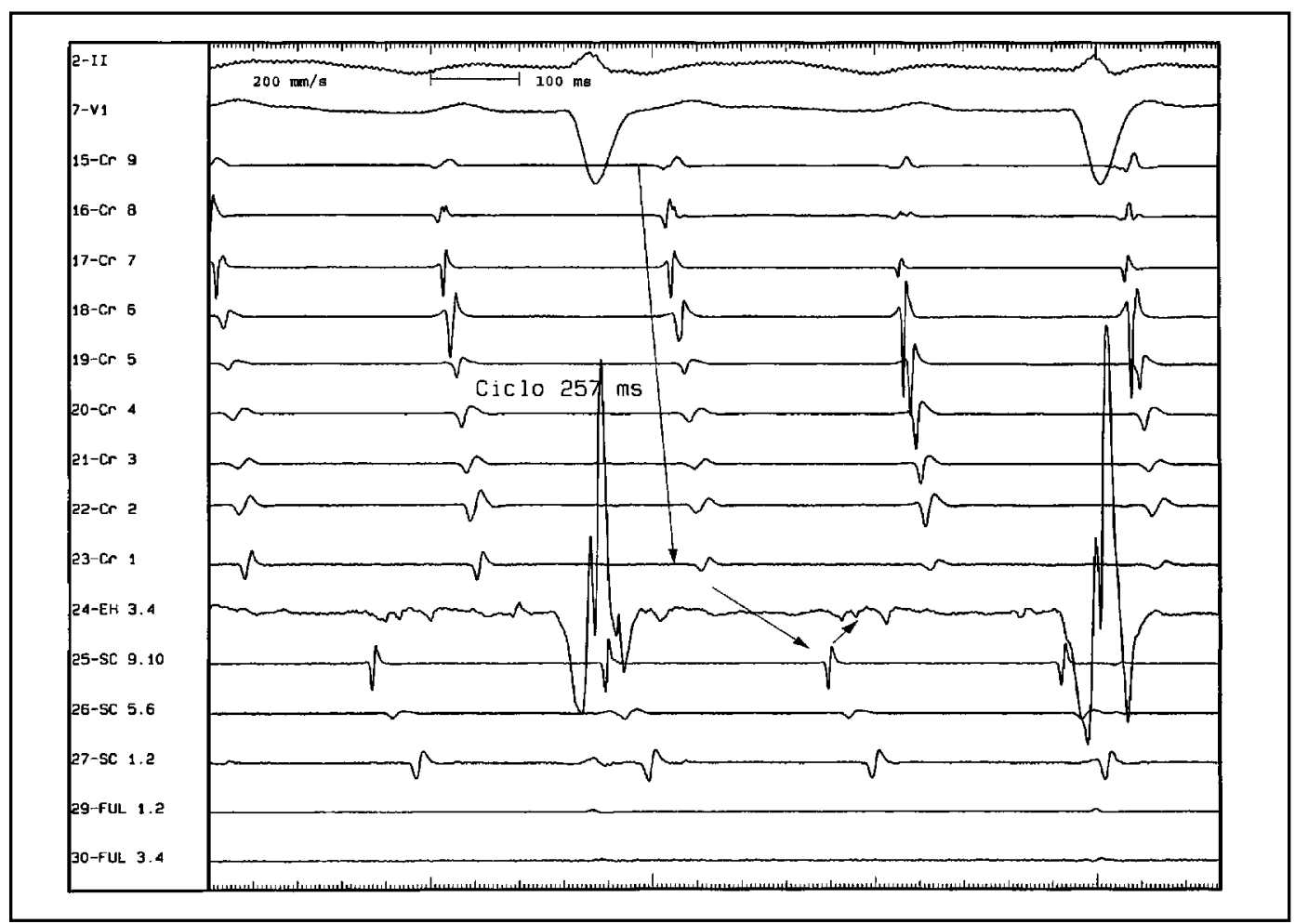

FIGURA 2. Registro endocavitario de un paciente con flutter auricular típico antihorario. Las flechas indican la dirección del circuito de la taquicardia. Las señales marcadas con $\mathrm{Cr}$ corresponden al registro con un catéter de 20 polos ubicado en la crista terminalis, con los electrodos distales $(\mathrm{Cr} 1)$ en la parte baja de la crista y los proximales ( $\mathrm{Cr} 9)$ en la crista alta. EH 3.4 es la señal del catéter cuadripolar ubicado a nivel del His y que registra la depolarización de la aurícula septal. Los electrogramas de los canales rotulados como SC muestran la activación de la aurícula izquierda: SC 9.10 es el par de electrodos más proximal, ubicado a nivel del os del seno coronario y SC 1.2 es el par de electrodos distal ubicado en la aurícula izquierda lateral. Se observa una señal auricular con ciclo de $257 \mathrm{~ms}$ (233 lpm) que baja a lo largo de aurícula derecha lateral hasta llegar a la crista distal ( $\mathrm{Cr} 1$ ), donde sufre un enlentecimiento que corresponde al paso del impulso a través del istmo cavo-tricuspídeo, para luego activar el os del seno coronario y subir por el septum interauricular (EH 3.4) hasta llegar nuevamente a la aurícula derecha alta y volver a bajar por la aurícula derecha lateral. 
mecanismo de la arritmia. En la práctica no realizamos entrainment, dada la dificultad técnica y la prolongación de tiempos en el EEF. La participación del ICT en el circuito de la taquicardia se confirmó indirectamente por la interrupción de ésta durante $R F$ y la incapacidad de inducir otras arritmias post-RF.

Fulguración. Se utilizaron catéteres de fulguración EPT con punta de $4 \mathrm{~mm}$ (8 French) o de $10 \mathrm{~mm}$ (8 French). Se aplicó RF con un equipo EPT-1000XP, con el propósito de crear una línea de bloqueo bidireccional de la conducción desde el anillo tricuspídeo hasta la vena cava inferior, utilizando temperatura de 50 a $70^{\circ} \mathrm{C}$ y energía de 50 Watts.
El procedimiento se realizó con el paciente en FL o en ritmo sinusal. La aplicación de RF durante FL resultó en la interrupción de éste. La aplicación de RF durante ritmo sinusal, se realizó durante estimulación auricular desde seno coronario de modo de documentar cambio en la secuencia de activación de la crista terminalis (Figura 3). Además, se mapeó a lo largo del ICT durante estimulación desde seno coronario, para descartar la presencia de potenciales residuales, expresados por electrogramas auriculares fusionados.

Los pacientes se mantuvieron en observación por treinta minutos y se repitió la estimulación del seno coronario para comprobar persistencia del

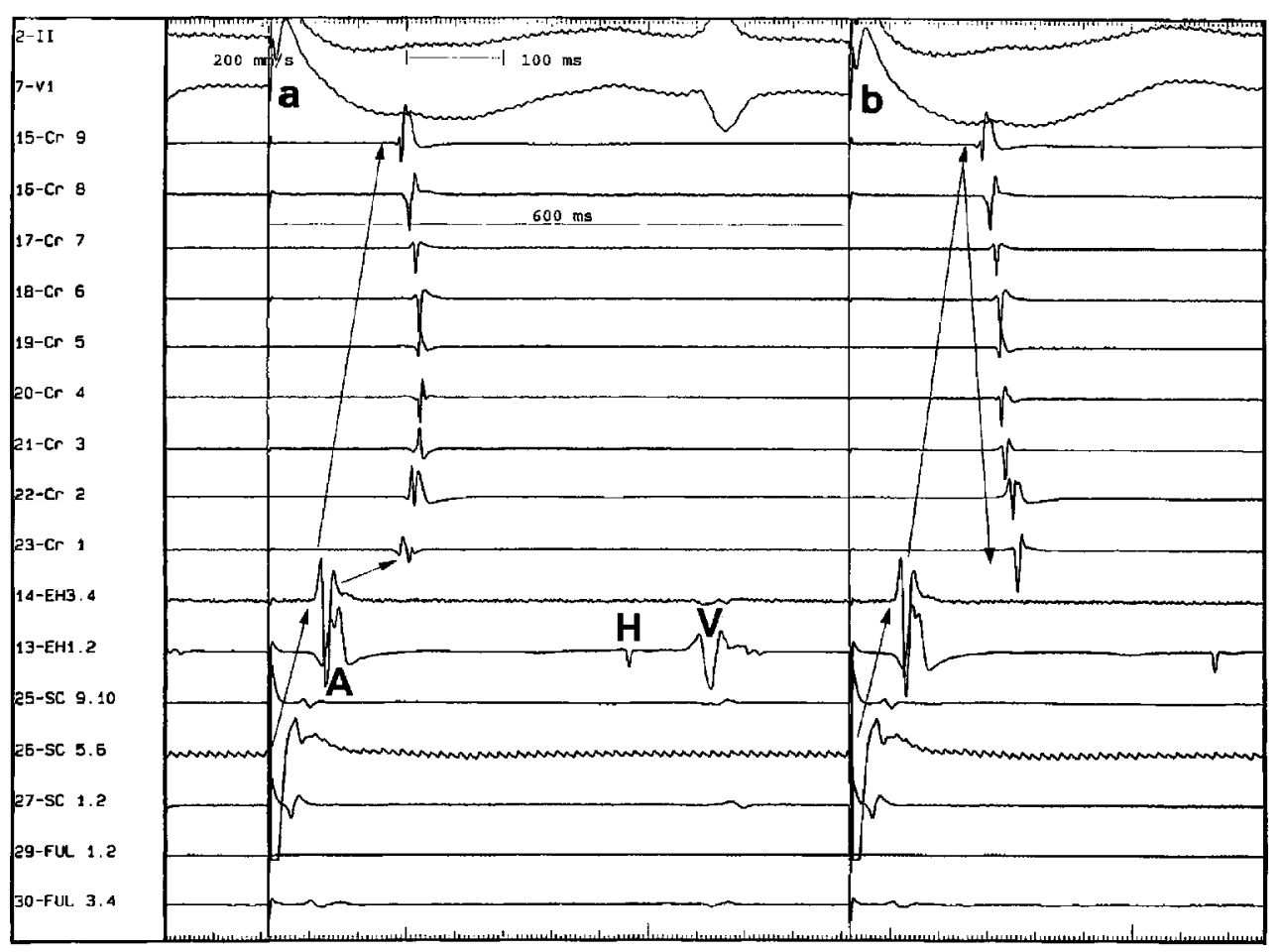

FiguRA 3. Señales endocavitarias durante aplicación de radiofrecuencia en el istmo cavo-tricuspídeo. Abreviaciones como en la Figura 2. Se muestran dos latidos consecutivos mientras se estimula la aurícula desde seno coronario (SC). En "a" el impulso se propaga desde el sitio de estimulación SC 5.6 llegando primero al septum interauricular (EH 3.4) y desde ahí sube hasta alcanzar la aurícula derecha alta (Cr 9) y baja por la pared lateral. Al mismo tiempo, desde el septum bajo el impulso se propaga a través del istmo cavo-tricuspídeo y logra activar la región inferior de la cara lateral de la aurícula derecha (Cr 1) resultando en activación fusionada de la crista terminalis. En "b", la secuencia de activación de la crista cambia, en este caso el impulso no atraviesa el istmo cavo-tricuspídeo por lo que no llega precozmente a la crista distal. De este modo la crista se activa solamente en sentido céfalo-caudal (de $\operatorname{Cr} 9$ a $\operatorname{Cr} 1$ ). Esto debido a que ha ocurrido bloqueo de la conducción a nivel del istmo cavo-tricuspídeo. 
bloqueo del ICT y la no reinducción de FL, sin usar isoproterenol.

Seguimiento. Se contactó telefónicamente a los pacientes sometidos a fulguración por FL en nuestro laboratorio y se les citó para evaluación clínica y electrocardiográfica.

\section{RESULTADOs}

Características de los pacientes. De los 86 pacientes, 5 habían fallecido por causa no atribuible a su arritmia y 5 no pudieron ser contactados.

De los 76 pacientes restantes, la edad promedio fue de 56,3 años (21-77) y 58 (56\%) eran hombres. En 39 pacientes existía antecedente de otra cardiopatía (Tabla 1).

Todos los pacientes eran sintomáticos por palpitaciones, 19 presentaban además disnea y 3 tenían historia de pre-síncope. Un paciente debutó con insuficiencia cardíaca, sospechándose taquicardiomiopatía.

El FL era paroxístico en 63 pacientes (83\%) y crónico en 13 (17\%). Todos tenían FL típico antihorario. Sesenta y cuatro pacientes (84\%) estaban con tratamiento antiarrítmico, de los cuales 22 recibían más de uno. Además, 25 pacientes (33\%) recibían anticoagulación oral. En 21 pacientes $(28 \%)$ se había realizado cardioversión eléctrica externa en el manejo de su flutter.

Respecto al antecedente de arritmias, 4 pacientes con FL habían sido sometidos a fulguración con RF del ICT previamente, 2 eran portadores de displasia arritmogénica del ventrículo derecho con taquicardia ventricular demostrada y fulgurada $\mathrm{y}$,

\section{Tabla 1. Tipos de cardiopatía estructural en los pacientes derivados para fulguración del istmo cavo-tricuspídeo por flutter auricular típico ( $\mathrm{N}=39$ )}

\begin{tabular}{|lr|}
\hline Tipo de cardiopatía estructural & $\mathrm{N}^{\circ}$ pacientes \\
\hline Cardiopatía hipertensiva & 19 \\
Comunicación interauricular operada & 7 \\
Cardiopatía coronaria & 4 \\
Enfermedad valvular & 4 \\
Otro & 5 \\
\hline
\end{tabular}

28 pacientes (37\%) tenían fibrilación auricular (FA) documentada. De los pacientes con FA, 46\% tenía antecedente de cardiopatía asociada, principalmente cardiopatía hipertensiva (Figura 4).

EEF y fulguración. Al momento del ingreso al laboratorio, 45 pacientes (59\%) estaban en FL típico antihorario, 29 en ritmo sinusal y 2 en FA; estos 2 pacientes fueron cardiovertidos eléctricamente previo a la fulguración del ICT.

Se logró fulguración exitosa del ICT en los 76 pacientes sometidos al procedimiento, confirmada por la persistencia de bloqueo bidireccional en el ICT durante estimulación de seno coronario.

La única complicación asociada al procedimiento fue bloqueo aurículo-ventricular de alto grado secundario a la $\mathrm{RF}$ en 1 paciente, portador de displasia aritmogénica de ventrículo derecho con implante previo de desfibrilador automático unicameral.

El tiempo total del procedimiento fluctuó entre 2,5 y 4,5 horas y el de fluoroscopía entre 20 y 50 minutos.

Todos los pacientes fueron dados de alta en ritmo sinusal documentado por electrocardiograma.

Seguimiento. El tiempo promedio de seguimiento fue de 20,1 meses (3-40). A todos los pacientes se les realizó control clínico y electrocardiograma. En aquéllos con síntomas sugerentes de probable recidiva de arritmia, se complementó la evaluación con Holter de arritmias de $24 \mathrm{~h}$.

Al momento de la evaluación, 62 pacientes (82\%) se encontraban en ritmo sinusal (3 de ellos

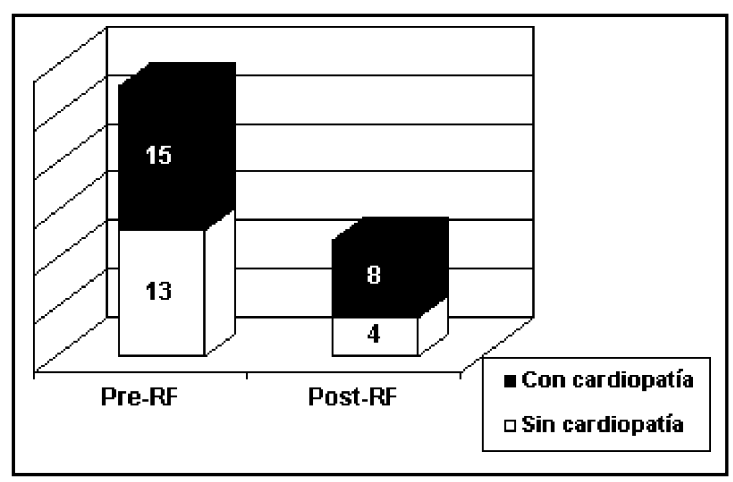

Figura 4. Pacientes con fibrilación auricular antes y después de fulgurar el istmo cavo-tricuspídeo según presencia o no de cardiopatía estructural. 
con marcapaso definitivo), 12 (16\%) en FA y 2 (2\%) en FL atípico. De los 12 pacientes con FA, 6 habían presentado FA antes de la fulguración con $\mathrm{RF}$ y 6 debutaron con FA post fulguración. Durante el seguimiento, 2 pacientes $(2.6 \%)$ requirieron implante de marcapaso definitivo por enfermedad del nódulo sinusal (Figura 5).

De los pacientes con FA, 8 (66\%) tenían cardiopatía asociada (Figura 4).

La mayoría de los pacientes refiere mejoría significativa en su calidad de vida, persistiendo con síntomas, principalmente palpitaciones, sólo aquéllos con documentación de arritmia, ya sea recidiva del FL o aparición de FA.

\section{Discusión}

El manejo médico farmacológico del FL es complejo, dado una baja tasa de conversión a ritmo sinusal, alta frecuencia de recidiva y difícil control de la frecuencia ventricular. Además, los antiarrítmicos tienen efectos secundarios entre los que se incluye la proarritmia. Inicialmente se consideró la $\mathrm{RF}$ como una opción ante el fracaso del tratamiento médico. Superada la curva de aprendizaje y con el diseño de nuevos catéteres y de vainas especializadas, aumentó el porcentaje de éxito y disminuyeron a un mínimo las complicaciones. Debido a ello, pensamos que en la actualidad el tratamiento con $\mathrm{RF}$ ha reemplazado al farmacológico como primera elección en los casos de FL típico, ya sea primer episodio o recidiva de éste. Este pensamiento es compartido por otros grupos ${ }^{6-8}$.

$\mathrm{Si}$ bien la incidencia exacta del FL no se conoce, se sabe que $10 \%$ de los pacientes que se presentan con taquicardia supraventricular corresponden a FL y que éste es más frecuente en hombres ${ }^{9,10}$, tal como ocurrió en la presente serie.

Se ha descrito que una alta proporción de pacientes con FL también padece de FA o cardiopatía estructural. En nuestra serie, previo a la fulguración del ICT, 28 pacientes (37\%) tenían FA además de FL Al final del seguimiento, sólo 12 pacientes (16\%) se encontraban en FA. De estos 12 pacientes, 8 presentaban factores de riesgo para desarmollar FA (cardiopatía hipertensiva o coronaria, miocardiopatía dilatada y limitación crónica al flujo aéreo), por lo cual no es de extrañar que esta se mantuviese post RF. Los 4 pacientes restantes que se mantuvie-

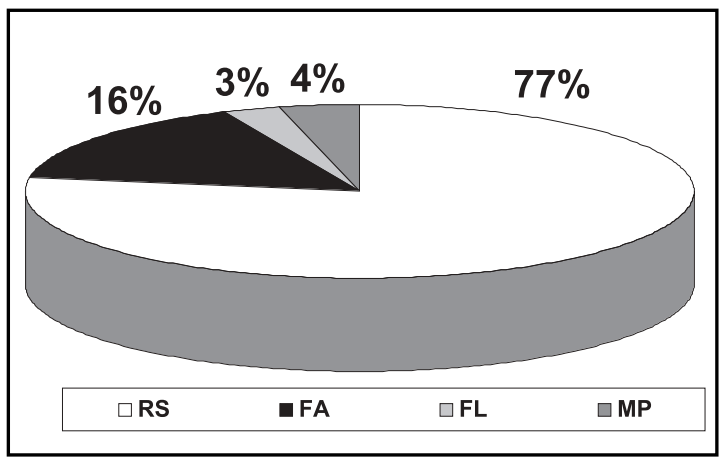

Figura 5. Ritmo cardíaco al momento del seguimiento de los pacientes sometidos a fulguración del istmo cavo-tricuspídeo por flutter auricular. RS: ritmo sinusal. FA: fibrilación auricular. FL: flutter auricular. MP: marcapaso.

ron en FA, no tienen una causa evidente, ya que carecían de cardiopatía. Es probable que en ellos la FA sea la manifestación inicial de un trastorno eléctrico auricular independiente del flutter, cuya evolución natural sería hacia el establecimiento permanente de FA.

Por lo tanto, nuestros resultados documentan una disminución de la prevalencia de FA en pacientes sometidos a RF de FL: 16 pacientes dejaron de tener FA post RF de FL Otros autores han reportado resultados similares ${ }^{11-13}$. Si bien el tratamiento con RF del FL no es un tratamiento que sane la FA de todos aquellos pacientes que la tienen concomitancia de ambas arritmias, en nuestra experiencia un porcentaje elevado de pacientes con FA y FL, disminuyen la prevalencia de FA al fulgurar el FL. Es probable que el FL contribuya a mantener la FA a través de varios mecanismos. Entre estos se puede mencionar: la estimulación a alta frecuencia a que es sometida la aurícula izquierda durante flutter auricular derecho y el remodelamiento de las aurículas con acortamiento de los períodos refractarios de éstas. El FL induce FA, como consecuencia de la estimulación auricular rápida, similar a lo que ocurre en el laboratorio de electrofisiología cuando se induce FA con estimulación auricular rápida. La fulguración del FL puede producir normalización de la refractariedad auricular y regresión del remodelamiento eléctrico, con lo cual se disminuye la posibilidad de FA ${ }^{14-16}$. Por este motivo, se recomienda la fulguración del ICT en 
pacientes con FL y FA basados en la alta tasa de éxito y en el impacto sobre la recurrencia y posiblemente severidad de la FA.

El tratamiento actual, que consiste en bloquear la conducción bidireccional a través del ICT mediante fulguración con $\mathrm{RF}$, ofrece un alto porcentaje de éxito. Los pacientes recuperan ritmo sinusal, con bajo porcentaje de complicaciones ${ }^{17-}$ 20. En vista de la alta eficacia y malos resultados del manejo médico convencional del FL, la fulguración con $\mathrm{RF}$ debe ser considerada como terapia de primera línea en pacientes con FL típico.

\section{LIMITACIONES}

El estudio está constituido por una muestra heterogénea de pacientes, en la que se incluyen

\section{REFERENCIAS}

1. Jais $\mathrm{P}$, Shah D, Haissaguerre M, Hocini M, Peng J, TAKAHASHI A ET AL. Mapping and ablation of left atrial flutters. Circulation 2000; 101: 2928-34.

2. Olgin JE, Jayachandran JV, Engesstein E, Groh W, ZIPES D. Atrial macroreentry involving the myocardium of the coronary sinus: a unique mechanism for atypical flutter. J Cardiovasc Electrophysiol 1998; 9: 1094-9.

3. Schwartzman D, Calians DJ, Gottlieb CD. Conduction block in the inferior vena caval-tricuspid valve isthmus: association with outcome of radiofrequency ablation of type I atrial flutter. J Am Coll Cardiol 1996; 28: 1519-31.

4. Cosio FG, Lopez-Gil M, Goicolea A. Radiofrequency ablation of the inferior vena cava-tricuspid valve isthmus in common atrial flutter. Am J Cardiol 1993; 71: 705-9.

5. García R, Ruiz R, Morell S, Sanuan R, Martínez J, Вотеша S. Flutter Auricular, En: Electrofisiología Cardiaca Clínica y Ablación. España: Editorial McGraw - Hill Interamericana de España, S.A.U., 1999; 287-303.

6. Fischer B, HaissaguerRe M, GarRigues S. Rediofrequency catheter ablation of common atrial flutter in 80 patients. J Am Coll Cardiol 1995; 25: 136572. pacientes consecutivos referidos para fulguración con RF del ICT con y sin cardiopatía estructural y no se uniformó el tratamiento que recibieron prefulguración.

No se realizó Holter de arritmias y ecocardiograma en forma sistemática pre y post-procedimiento. Por este último motivo, en algunos pacientes el diagnóstico de cardiopatía estructural, sobre todo los hipertensos, probablemente no sea certero.

El uso de antiarrítmicos y la indicación de anticoagulación post-fulguración con RF del ICT quedó a criterio del médico tratante, por lo cual no existe homogeneidad.

En algunos pacientes, el seguimiento aún es breve, pero los resultados obtenidos muestran que la interrupción del circuito del FL es un procedimiento con alto porcentaje de éxito y muy bajas tasas de complicaciones.

7. Poty $\mathrm{H}$, Saoudi N, Abdel Aziz A. Radiofrequency catheter ablation of type I atrial flutter: prediction of late success by electrophysiologic criteria. Circulation 1995; 92: 1389-92.

8. FeLD GK, FLeCK R, Chen P. Radiofrequency catheter ablation for the treatment of human type I atrial flutter: identification of a critical zone in the reentrant circuit by endocardial mapping techniques. Circulation 1992; 86: 1233-40.

9. Bialy D, Lehman MH. Hospitalization for arrhythmias in the United States: importance of atrial fibrillation. J Am Coll Cardiol 1992; 19:716.

10. Weliens H. Contemporary Management of Atrial Flutter. Circulation 2002; 106: 649-52.

11. Paydack H, Kall JG, Burke M. Atrial fibrillation after radiofrequency ablation of type I atrial flutter: time to onset determinants and clinical course. Circulation 1998; 98: 315-22.

12. NABAR A, Rodriguez LM, Timmermans C. Effect of the right atrial isthmus ablation on the occurrence of atrial fibrillation. Observations in 4 patient groups having type I atrial flutter with or without associated atrial fibrillation. Circulation 1999; 99: 1441-5.

13. TAI CT, Chen SA, Chiang CE. Long term outcome of radiofrequency catheter ablation for typical atrial flutter: risk prediction of recurrent arrhythmias. J Cardiovasc Electrophysiol 1998; 115:21. 
14. ZIPES D. Electrophysiological remodeling of the heart owing to rate. Circulation 1997; 95: 1745-8.

15. Jordaens L, Missault L, Germonpré E, Caluens B, Adang L, Vandenbogaerde J et al. Delayed restoration of atrial function after conversion of atrial flutter by pacing or electrical cardioversion. Am J Cardiol 1993; 71: 63-7.

16. Ausma J, Wijfrels M, Thoné F, Wouters L, Auessie M, BORgERS M. Structural changes of atrial myocardium dueto sustained atrial fibrillation in the goat. Circulation 1997; 96: 3157-63.

17. Cosio FG, López-Gil M, Arribas F, Goicolea A, PAstor A, NúÑEz A. Ablación de flutter auricular. Resultados a largo plazo tras 8 años de experiencia. Rev Esp Cardiol 1998; 51: 832-9.
18. LeE S, TaI C, Yu W, Chen Y, Hsieh M, Tsai C. Effects of radiofrequency catheter ablation on quality of life in patients with atrial flutter. Am J Cardiol 1999; 84: 278-83.

19. Natale A, Newby K, Pisano E, Leonelu F, Fanem R, Potenza D. Prospective randomized comparison of antiarrhythmic therapy versus first line radiofrequency ablation in patients with atrial flutter. J Am Coll Cardiol 2000; 35: 1898-904.

20. O'Callaghan P, Meara M, Kongsgaard E, PoloNiecki J, Luddington L, Foran J et al. Symptomatic improvement after radiofrequency catheter ablation for typical atrial flutter. Heart 2001; 86: $167-71$. 